\title{
Viewpoint
}

\section{Particle assembly from fluids}

\author{
Howard A. Stone \\ Department of Mechanical and Aerospace Engineering, Princeton University, Princeton, NJ 08544, \\ USA
}

Published February 28, 2011

Advances in the technology of microfluidics should make it possible to assemble complex structures out of individual particles.

Subject Areas: Fluid Dynamics

A Viewpoint on:
Algorithm for a Microfluidic Assembly Line
Tobias M. Schneider, Shreyas Mandre and Michael P. Brenner
Phys. Rev. Lett. 106, 094503 (2011) - Published February 28, 2011

Microfluidic technologies, which refers to a wide class of methods for controlling fluid flows at the scale of a few hundred microns and smaller, are leading to new approaches for addressing biophysical and biochemical problems at the scale of individual cells and innovative uses of droplets, bubbles, and small particles. Most applications for microfluidics are focused on channeling or screening miniscule amounts of liquid and whatever chemicals or particles are carried within it. But, the same technology that allows us to control liquids on the micron scale can be helpful in the assembly of complex objects out of smaller components. This is the idea behind a proposal for a microfluidic assembly device that Tobias Schneider, Shreyas Mandre, and Michael Brenner at Harvard University present in a paper appearing in Physical Review Letters [1]. They propose basic principles, supported by numerical simulations, for using fluid motion to control the trajectories of individual particles so as to assemble the particles into more complicated aggregates.

One route to organizing complex structures out of micron-scale particles is to specially design each component to recognize its mate (or mates) through selective interactions and assemble them piece by piece. For example, particles floating at a liquid-air interface can selfassemble due to capillary forces (a "capillary bond"). Here, the discrete control comes from spatially patterning different wettabilities over the surface of the particles [2]. Alternatively, thermal fluctuations can promote the binding of colloid particles containing "programmed" DNA strands [3] or the interlocking of appropriately shaped "lock-and-key" colloids [4]. Finally, small numbers of particles in close proximity can be manipulated with electric fields [5] and laser tweezers [6].

In their work Schneider et al. explore the possibility of using the force of moving fluid to bring two or many particles together into a static configuration. They

DOI: $10.1103 /$ Physics .4 .17

URL: http://link.aps.org/doi/10.1103/Physics .4.17
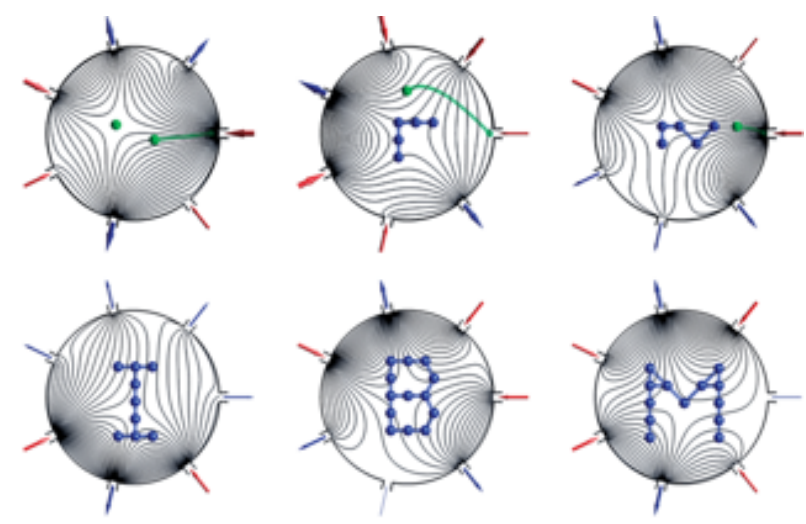

FIG. 1: Flow-driven assembly, one particle at a time, forms distinct letters. (Credit: Schneider et al. [1])

consider a flat chamber- somewhat like a closed petri dish-into or out of which fluid can flow through evenly spaced inlets along the rim (Fig. 1). The flow rates of the inlets can be independently controlled.

Particles introduced at the various inlets are then manipulated by the flow, and when the particles get close together, the authors assume they are irreversibly bound together, as can be achieved by chemical means. With this simple model in mind, Schneider et al. ask: How many flow rates are needed to manipulate $N$ particles?

By design, the team focuses their attention on microfluidic configurations where inertial effects are typically small. This "low Reynolds number" approximation is central to their mathematical characterization of the fluid dynamics. The fluid motion in this limit depends linearly on all of the "knobs" available for generating and manipulating the flow through the inlets. In this picture, if there are no external forces on the individual particles, they tend to move with the local fluid

(c) 2011 American Physical Society 
velocity, which in turn can be regulated by the flow rates specified at various entrances to and exits from the microfluidic device.

Suppose the goal is to organize $N$ particles by manipulating all of them simultaneously. In one algorithm the particle trajectories can be assumed as given and it is necessary to solve for the flow rates at each inlet that make specified particle trajectories possible. Schneider et al. find that this approach, while feasible for a few particles, cannot manipulate a large number of particles.

Instead, they propose a sequential algorithm where the particles are assembled one at a time into a larger organized aggregate. To understand how a finite number of controls (i.e., the flow rates at the boundary inlets), in principle, allow a sequential algorithm for assembly, consider the two-dimensional case. To control the position of the large aggregate requires specifying two coordinates, i.e., this step corresponds to two degrees of freedom. The location of the free particle to be added to the aggregate also requires two degrees of freedom. Control of the aggregate in order to properly locate the free particle at a specified position around the periphery requires creating a stagnation point in the flow about the aggregate (technically, this step requires a velocity field that varies linearly with position measured about the aggregate), which involves the orientation of the flow and its magnitude, i.e., there are two more degrees of freedom. Finally, the system must conserve the fluid volume so the controlled flow rates must add to zero. Thus, there are seven degrees of freedom and consequently seven inlets or flow rates are required. The same arguments applied to the three-dimensional case suggest that 11 different flow rates are required to sequentially structure an aggregate.

What kinds of two-dimensional assemblies might be possible with a fluidic manipulator like this? As one example among many possibilities, Schneider et al. give an instructional demonstration where letters of the alphabet are organized by manipulating independent particles and assembling them into recognizable shapes. In this way, they show their sequential algorithm can organize letters such as I, B, and M (Fig. 1). Consequently, they suggest that in two dimensions these ideas should allow the construction of arbitrarily shaped aggregates.

The authors recognize that there may be limitations to this approach, and some of the limitations may prove daunting. For example, for micron dimension particles (or smaller) Brownian motion is important and such thermal noise may prove to be a significant barrier if the trajectories of the particles are highly sensitive. The latter is possible since it is well known that coupled nonlinear ordinary differential equations, such as those that describe the particle dynamics in these systems, can be chaotic, meaning they are sensitive to small perturbations. Moreover, the Harvard group's model is based on a set of assumptions, each of which neglect certain physical effects, some of which are known to have systematic influences on particle motion in microfluidic systems. As but one example, small inertial effects can lead to positioning of small particles due to drift across streamlines [7]. Finally, in these confined systems, hydrodynamic interactions, i.e., the movement of one particle is influenced by a boundary or another particle, may create unforeseen difficulties. An example of this kind of instability plagues the stable formation of microfluidic crystals [8].

In spite of potential hurdles, the idea of microfluidic assembly, one particle at a time, suggests intriguing possibilities. Other particle-scale manipulation approaches fall into the same category. Imagine an assembly line where particles of different material type, size, and shape and different cells are available for particleby-particle manipulation into a complex aggregate. At the least, such an approach may make new kinds of biological assays possible, and at its most speculative, new kinds of machines may be possible.

\section{Acknowledgments}

The author thanks Dr. Bo Sun for helpful feedback.

\section{References}

[1] T. M. Schneider, S. Mandre, and M. P. Brenner, Phys. Rev. Lett. 106, 094503 (2011).

[2] N. Bowden, A. Terfort, J. Carbeck, and G. M. Whitesides, Science 276, 233 (1997).

[3] C. A. Mirkin, R. L. Letsinger, R. C. Mucic, and J. J. Storhoff, Nature 382, 607 (1996).

[4] S. Sacanna, W. T. M. Irvine, P. M. Chaikin, and D. J. Pine, Nature 464, 575 (2010).

[5] A. E. Cohen, Phys. Rev. Lett 94, 118102 (2005).

[6] D. R. Grier and Y. Roichman, Appl. Opt. 45, 880 (2006).

[7] D. Di Carlo, Lab Chip 9, 3038 (2009).

[8] T. Beatus, T. Tlusty, and R. Bar-Ziv, Nature Phys 2, 743 (2006). 


\section{About the Author}

\section{Howard A. Stone}

Howard A. Stone is the Donald R. Dixon '69 and Elizabeth W. Dixon Professor in Me-
chanical and Aerospace Engineering at Princeton University. He received his S.B. degree
in chemical engineering from the University of California, Davis, and a Ph.D. in chemical
engineering from Caltech. From 1989 to 2009 he was on the faculty in the School of En-
gineering and Applied Sciences at Harvard University. His research interests are in fluid
dynamics, especially as they arise in research and applications at the interface of engineer-
ing, chemistry, and physics. He was the first recipient of the G. K. Batchelor Prize in Fluid
Dynamics, which was awarded in August 2008. In 2009 he was elected to the National
Academy of Engineering. In 2008 he was recognized as an Outstanding Referee by the
American Physical Society.

